\title{
Experiencias relacionadas con la implementación de políticas de inclusión social en educación a distancia
}

\author{
Lía Margarita Jaraba Ortiz \\ Fonoaudióloga. \\ Especialista en Investigación Aplicada a la Educación. \\ Magíster en Neuropsicología y Educación. \\ Corporación Unificada de Educación Superior - CUN \\ Correo electrónico: liamargui@hotmail.com
}

Recibido: 06 de junio de 2015 - aprobado: 23 de agosto de 2015

\section{Cómo citar este artículo}

Jaraba, L. M. (2015). Experiencias relacionadas con la implementación de políticas de inclusión social en educación a distancia. Espiral. Revista de Docencia e Investigación, 5(2), 111-126.

\section{Resumen}

En el siguiente artículo se presenta una revisión bibliográfica con el propósito de identificar aquellos planteamientos teóricos fundamentales que sustentan la temática de la inclusión social en la educación superior por medio de la modalidad a distancia. Para ello se realizó una búsqueda de información agrupada en tres bloques, uno en lo relacionado con la educación inclusiva y la inclusión social, otro con las políticas que la sustentan en los diversos países de América Latina como México, Argentina, Brasil, Chile y finalmente Colombia y, por último, sobre la educación a distancia y el concepto de inclusión. En los resultados se evidencia que aunque se han realizado múltiples investigaciones y se han implementado políticas y estrategias, aún se hace necesario continuar propiciando ambientes universitarios capaces de apreciar y respetar las diferencias.

Palabras clave: Inclusión, inclusión social, inclusión educativa, educación superior, modalidad a distancia.

\footnotetext{
Abstract

In the following article, a theoretical framework is intended to identify those theoretical approaches that sustain the subject of social inclusion in post-secondary education through distance learning. For this, a data research was carried out and grouped into three blocks: the first one related to inclusive education and social inclusion. The second one related to the politics that support them in all the countries of Latin America as Mexico, Argentina, Brazil, Chile and finally Colombia. The third one is about the distance learning and the definition of inclusion. It could be determined that even though there have been several politics research and strategies it is mandatory to keep creating educational environment in which people can appreciate and respect the differences.
}

Keywords: Inclusion, social inclusion, inclusive education, higher education, distance learning.

\section{Introducción}

Durante los últimos años el término inclusión social ha llegado a formar parte de las políticas gubernamentales de diversos países, incluyendo a América Latina. Es definido por Vivero (2012), como:

Un término igualmente polisémico y multifacético que la equidad y se define en función de las perspectivas y enfoques desde los cuales se aborde. Pero en todos los casos supone contener o englobar grupos sociales o personas dentro de espacios o circunstancias sociales específicos y está íntimamente relacionado con el término de exclusión social, como dos polos de un mismo eje en torno al cual se puede delimitar una gran variedad de situaciones de vulnerabilidad o precariedad social (párr. 3).

A pesar de la diversidad del término es fundamental su estudio y análisis dentro de las diferentes perspectivas y realidades sociales. En el área educativa, por ejemplo se puede entender como "una línea de acción que busca garantizar la igualdad de acceso a la ciencia, educación y cultura de algunos grupos sociales que han sido memorizados por distintas razones" (Vivero, 2012, párr. 4). 
En este sentido, se ha establecido una relación entre los términos educación e inclusión donde un aspecto se convierte en una estrategia para el otro, permitiendo visionar un modelo educativo abierto y capaz de entender la diversidad como inherente a la vida misma (MEN, 2013).

Así mismo, dentro de estos planteamientos se destaca el papel de las instituciones de educación superior, tomando en cuenta que dentro de las políticas y estrategias para incentivar la permanencia y graduación 2013-2014 se plantea como una necesidad la atención a la diversidad e inclusión de poblaciones, tales como grupos étnicos (indígenas, afrocolombianos), población con necesidades educativas especiales (en situación de discapacidad o con talentos excepcionales), aquellos afectados por la violencia (desplazados, víctimas de minas antipersona) y habitantes de frontera (MEN, 2013).

Sin embargo, aunque se ha ido estableciendo los lineamientos y políticas de inclusión educativa, según el MEN (2007) Colombia tiene altos niveles de inequidad y exclusión social, lo que contrasta con el gran número de etnia y diversidades que se presentan en el país y en lo relacionado con la discapacidad, 12 de cada 100 niños presentan dificultades que limitan su aprendizaje y solo 3 de estos asisten a la escuela.

De igual forma, el DANE (citado por MEN, 2013) plantea que el porcentaje de colombianos en situación con discapacidad que ingresa a la educación superior está por debajo del $1 \%$. Por lo cual, se hace urgente consolidar estrategias que permitan una verdadera educación inclusiva.

A continuación se presentará una revisión bibliográfica sobre la inclusión en la educación superior por medio de la modalidad a distancia, buscando identificar aquellos planteamientos teóricos fundamentales que sustenten esta temática. Esto se iniciará indagando sobre la inclusión social en las políticas de educación superior y luego en la modalidad a distancia, y así destacar la experiencia de países latinoamericanos como México, Argentina, Brasil, Chile y, finalmente Colombia.

\section{Materiales y métodos}

Para la realización de la presente revisión bibliográfica se partió de la búsqueda de información relacionada con la educación inclusiva y las políticas públicas de inclusión en la educación superior, teniendo en cuenta como criterio que fueran investigaciones realizadas en México, Argentina, Brasil, Chile y Colombia.

Además, se seleccionaron aquellos artículos científicos que desarrollarán la temática de la inclusión en la educación superior, especialmente, en la modalidad a distancia y que enfatizarán en grupos étnicos (indígenas, afrocolombianos), población con necesidades educativas especiales (en situación de discapacidad o con talentos excepcionales); población afectada por la violencia (desplazados, víctimas de minas antipersona) y habitantes de frontera.

Así mismo, se plantean como criterio de inclusión los artículos publicados entre el 2005 y 2015, utilizando buscadores, tales como: Google académico, Scielo, Dialnet y Redalyc.

\section{Resultados}

\section{La educación inclusiva y la inclusión social}

De acuerdo con lo planteado por Dueñas (2010)

El término inclusión educativa se define de múltiples formas, no existiendo un significado concreto y único del mismo, por lo que se utiliza para referirse a situaciones y fines diferentes y en contextos distintos. Es decir, no se limita al ámbito de la educación sino que se refiere a todos los ámbitos de la vida (p. 358).

Así mismo, Infante (2010) al hacer un análisis de la inclusión educativa considera que el concepto ha migrado desde la educación especial, permitiendo la construcción de políticas 


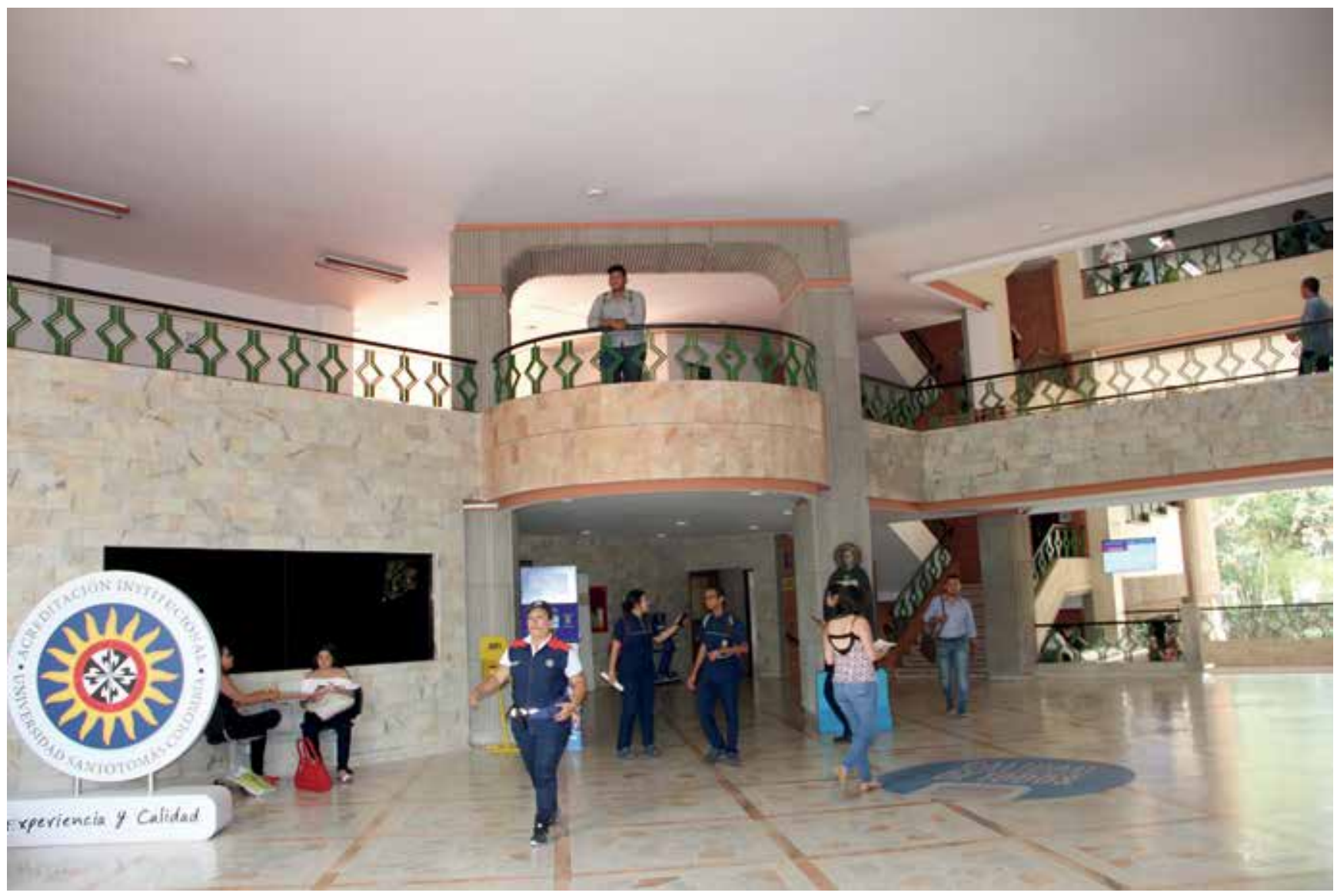

traducidas en diversas acciones, pero que debido a las concepciones y esquemas antiguos se producen dificultades, que deben abordarse desde la formación docente y la relación que se establece con la sociedad.

Es claro que educación y sociedad van de la mano, donde los cambios que se produzcan en una afectará en gran medida a la otra y por lo tanto:

La creciente toma de conciencia de la sociedad acerca de las desigualdades sociales y la progresiva consolidación de los derechos humanos, en particular el derecho a la educación, a la igualdad de oportunidades y a la diversidad, vienen propiciando en la sociedad actual, el surgimiento y uso del término inclusión (Dueñas, 2010, p. 361).

Ahora, al hablar de educación inclusiva es fundamental recordar que es una respuesta al derecho fundamental de toda persona y que por lo tanto se hace necesario que se fomente la equidad (Unesco, 1990), ante lo cual Juárez (2010) plantea que esta:

Acoge en su seno sin reservas a todos los candidatos independientemente de su origen social, cultural, etnia o capacidades físicas, brindándoles una educación de calidad para fortalecer las posibilidades sociales de equidad y oportunidades de desarrollo humano equivalentes. Esto significa que la escuela inclusiva brinda oportunidades de educación y desarrollo a todos los alumnos, los considerados normales y los considerados con necesidades educativas especiales, los que tienen recursos económicos y capitales intelectuales y sociales, como a aquellos que carecen de ellos (p. 71).

Por lo tanto, es clave entender que aunque la inclusión va más allá de lo educativo, pensar en educación es también reflexionar sobre la humanidad y sobre derechos humanos, implicando a la sociedad en general, pues ella es la que le debe dar a cada uno de sus miembros el 
lugar que necesitan y los elementos necesarios para saber desenvolverse, aprendiendo a respetar y aceptar las diferencias (Unesco, 2008).

Teniendo en cuenta lo anterior la política de educación superior inclusiva del MEN (2013) establece que esta se encuentra:

relacionada con la capacidad de potenciar y valorar la diversidad (entendiendo y protegiendo las particularidades), promover el respeto a ser diferente y garantizar la participación de la comunidad dentro de una estructura intercultural en los procesos educativos. Al trascender lo estrictamente académico y curricular para enfocarse en la constitución misma de lo social, la educación inclusiva tiene como objetivo central examinar las barreras para el aprendizaje y la participación propias de todo el sistema. En educación superior, no son los estudiantes los que deben cambiar para acceder, permanecer y graduarse: es el sistema mismo que debe transformarse para atender la riqueza implícita en la diversidad estudiantil (p. 5).

De esta forma, la educación inclusiva se convierte en una estrategia en contra de la exclusión, pero se diferencia de la inclusión educativa en que la primera identifica barreras para el aprendizaje, buscando su superación; mientras que la segunda parte de la adaptación de estudiantes al sistema (MEN, 2013).

Por lo anterior, al hablar de inclusión social se hace énfasis en la educación inclusiva, la cual debe tener como características fundamentales la participación, diversidad, interculturalidad, equidad y calidad.

En este sentido, se destacan varias experiencias de inclusión recopiladas por Mato (2008) en su texto sobre Diversidad Cultural e Interculturalidad en Educación Superior. Experiencias en América Latina, donde se observan proyectos realizados en diversas partes del continente y que aporta un panorama regional lleno de oportunidades pero también de problemas y retos al momento de abordar la inclusión.

\section{Inclusión social en las políticas de educación superior}

Al hablar de políticas de inclusión en educación superior es necesario partir del derecho fundamental a ella y que por lo tanto, cada país según su contexto establecerá los marcos legales que permitan un adecuado acceso, especialmente a las poblaciones más desfavorecidas.

Lo anterior, repercutirá en el diseño de currículos escolares y las formas de abordar el aprendizaje, con el propósito de asumir la diversidad como un potencializador de riquezas, en donde se atienden las diferencias de cada individuo pero también el gran aporte que puede brindarle a la sociedad (Unesco, 2000).

Ahora, en cuanto a que la educación sea un derecho Padilla (2011) plantea que debe tenerse en cuenta no solo para la inclusión de personas con discapacidad sino también para toda población que se sienta marginada, abarcando aspectos como la calidad y la permanencia que debe ser garantizada independiente de las condiciones físicas, sociales, emocionales o limitaciones del entorno.

Respecto a estas políticas de inclusión en la educación superior, Chiroleu (2009) expone que en América Latina se ha incrementado el interés sobre la temática debido a la gran desigualdad que caracteriza a la región siendo urgente la implementación de estrategias y mecanismos que faciliten una educación para todos.

A continuación se expondrán las principales políticas de inclusión en la educación superior de algunos países latinoamericanos tales como México, Argentina, Brasil, Chile y finalmente Colombia.

En el caso de México, se parte del hecho de que la constitución política en sus primeros artículos hace referencia a los derechos humanos, garantizando equidad para todos a nivel de educación, género, etnia entre otros; por lo 
cual el estado asigna un presupuesto para promover la igualdad de oportunidades, se crean los reglamentos y normas pertinentes para esto y los programas que involucren a las poblaciones vulnerables (Alcántara \& Navarrete, 2013).

Así mismo, para los mexicanos se partió del Programa Nacional de Fortalecimiento de la Educación Especial buscando una mayor formación de los maestros y un compromiso de las familias, aumentando además la cobertura de esta población (Suárez, Salinas \& Garnique, 2010).

Revisando las acciones emprendidas por el Estado en la década, según datos del "Programa Nacional para el Desarrollo de las Personas con Discapacidad 2009-2012", se ve efectivamente un avance significativo en atención a los niños y niñas con NEE, como se aprecia en el aumento de unidades de servicio de educación especial en funcionamiento (Pronaddis, citado por Suárez, Salinas \& Garnique, 2010, p. 56).

En este mismo sentido, Río (2015) plantea que en México se ha hecho énfasis en cuatro aspectos fundamentales, la reforma integral para la educación media superior (RIEMS), la creación de la universidad abierta y a distancia como una modalidad que amplía la oferta educativa, el programa Nacional de becas de excelencia académica en Educación media superior (PNBEAEMS) y por último, la definición de lineamientos y normativas que promulga la necesidad de constituir una comisión interdisciplinaria que apoye la diversidad en cada universidad.

De igual forma, Silas (citado por Alcántara \& Navarrete, 2013) plantea que la inclusión social en México también se aborda desde Programas dirigidos a grupos étnicos y minorías que cursan la educación superior, lo cual ha sido abordado desde dos perspectivas, uno encaminado a lo individual y otro, a lo comunitario; creando los programas de Apoyo a Estudiantes Indígenas de Educación Superior (PAEIIES) y el Programa México Nación Multicultural (Universidad Nacional Autónoma de México, UNAM).
Por su parte en Argentina, Chiroleu (2009) expone que se debe considerar como criterios de inclusión el ingreso directo a las instituciones de Educación superior y la gratuidad; pero además se destacan el Programa Nacional de Becas Universitarias (PNBU) y el Programa de Créditos para la Educación Superior, en donde además se plantea que

Las becas suponen una política inclusiva que procura reducir las dificultades económicas de jóvenes provenientes de sectores sociales desfavorecidos. EI PNBU prevé estrategias de acompañamiento de los becarios en su trayecto en la educación superior, a través de sistema de tutorías, cursos de nivelación, etc. Esto supone una preocupación por actuar sobre las variables que generan disímiles posibilidades de permanencia y egreso (Chiroleu, 2012, p. 296).

De igual forma, García (2014) expone que las tutorías al igual que las becas se convierten en una política para la inclusión y permanencia pero se hace necesario garantizar el acceso laboral una vez se culmine la etapa educativa.

En cuanto a Brasil, Chiroleu (2009) destaca algunos mecanismos como el apoyo económico a población vulnerable, políticas de acciones para combatir la discriminación y los cursos especiales. Pero específicamente para la educación superior se destaca el sistema vestibular, que coexiste con el de evaluación seriada de la Enseñanza Media o los resultados obtenidos en el Examen Nacional y también el otorgar becas privilegiando por raza y etnia. Además,

Se han encarado programas de expansión de la oferta pública y a partir de 2007 se establece el Programa de Reestructuración y Expansión de las Universidades Federales (REUNI) el cual tiene entre otros objetivos la creación de condiciones para la expansión de las vacantes en estas instituciones. En este sentido si se inscribe en el Plan de Desarrollo de la Educación presentado por el gobierno ese mismo año y se articula con el Plan Nacional de Educación del año 2001 ... Las universidades federales deben presentar proyectos que contemplen especialmente tres aspectos: 
flexibilidad curricular, apoyo para la formación pedagógica de los docentes que permita el desarrollo de prácticas pedagógicas modernas y el uso de tecnología y el desarrollo de mecanismos de inclusión que permitan alcanzar la igualdad de oportunidades en el acceso y la permanencia en las instituciones (MEC citado por Chiroleu, 2009, p. 10)

En Chile, se encuentran programas de ayudas estudiantiles para sectores vulnerables como personas con escasos recursos, víctimas de violación a los derechos humanos, grupos étnicos y minorías, además se plantea que aunque no hay algo específico para género no se evidencia diferencia que permitan pensar en discriminación hacia la mujer (RIAIPE, 2013).

En general, se destaca la investigación realizada por Pedroza \& Villalobos (2009) sobre políticas compensatorias para la equidad de la educación superior en Argentina, Bolivia y Venezuela, en donde se plantea que se prioriza la atención para grupos como indígenas, afrodescendientes, discapacitados y mujeres, tratando de superar los problemas de inequidad, aunque persistan por causas económicas o sociales.

También se encuentra la recopilación de experiencias presentada en la V Jornada de Cooperación Educativa con Iberoamérica sobre educación especial, destacándose el uso de nuevas tecnologías inclusivas en Chile y México, el programa maestro de integración laboral de Argentina, el de educación inclusiva de Brasil y el énfasis que en Colombia se le ha dado a la inclusión en el plan de formación de formadores (Narvete \& Blanco, 2010).

Ahora en lo que se refiere a Colombia, se parte del hecho de que la Constitución de 1991 protege a las personas con discapacidad señalando que "se dará protección para las personas con disminución física, sensorial, psíquica" (artículo 47) y "es obligación del Estado la erradicación del analfabetismo y la educación de personas con limitaciones físicas o mentales o con capacidades excepcionales" (artículo 68) y a partir de ella

Se generan, entonces, leyes, acuerdos, decretos y resoluciones que se pueden condensar así: en el aspecto educativo, y como normas generales para el acceso a la educación, aparecen la ley 115 de 1994 y la ley 361 de 1997, en las cuales se resalta la igualdad de oportunidades para la población con discapacidad. Entre otros documentos que deben ser considerados están el del Consejo Nacional de Política Económica y Social (CONPES) 2761, de enero 25, de 1995 como una política de prevención y atención a la discapacidad; 3 la ley 324 de 1996, por la cual se establecen normas a favor de la población sorda; el decreto 2082 de 1996, por el cual se reglamenta la atención educativa para personas con limitaciones o con capacidades o talentos excepcionales4 y la ley 361 del 7 de febrero de 1997, que establece mecanismos que favorezcan la integración de las personas con discapacidad (Molina, 2010, p. 4).

Así mismo, el MEN (2013) ha planteado los lineamientos y diversas estrategias de la política de educación superior inclusiva, estableciendo los principios de integralidad y flexibilidad, determinando como retos generar procesos académicos inclusivos, tener docentes capacitados, promover espacios de investigación, innovación y creación artística y cultural, sostener el proceso por medio de una estructura administrativa y financiera.

De igual forma, se ha priorizado en la educación inclusiva a grupos particulares tales como personas con discapacidad, grupos étnicos, población víctima, y habitante de frontera, abordando diversas estrategias que "dan cuenta en la mayoría de los casos de los dos elementos del principio de integralidad, es decir de la pertinencia y la calidad, así como del acceso, permanencia y graduación de los estudiantes" (MEN, 2013, p. 43).

De acuerdo con lo anterior, se evidencia que los países de la región han implementado diversas políticas, acuerdos y estrategias en el 
marco de la inclusión, notándose avances especialmente en lo relacionado con personas discapacitadas y por género (Chiroleu, 2009).

Sin embargo, Aponte (2008) plantea que los logros no han sido tan significativos y la región presenta un alta tendencia a la desigualdad, la marginación y a la pobreza, reflejada a su vez en las dificultades para el acceso, especialmente de la educación superior, de aquellos grupos socialmente desfavorecidos por cuestiones étnicas, económicas y raciales.

También, Viveros (2012) destaca que en materia de inclusión la mayoría de jóvenes afrodescendientes e indígenas no acceden a la educación superior y solo un $5 \%$ de la población que egresa de la universidad continúa estudios de postgrado. Así mismo, entre los diversos programas y facultades existente diferencias notorias en cuestión de género evidenciándose que solo "un 5.6\% de mujeres en ingeniería mecánica, y al
$76 \%$ y $80 \%$ en carreras de la Facultad de medicina como la fisioterapia y la fonoaudiología respectivamente, y de la Facultad de Ciencias Humanas como Trabajo Social con el 75.5\%" (Arango \& Munévar, citado por Viveros, 2012, p. 7).

En cuestión de etnia también se nota diferencias significativas, donde

Solo $4 \%$ de la población afrocolombiana consigue este nivel y solo un $7.07 \%$ de los estudiantes de postgrado son afrocolombianos. Por otra parte, el reducido número de hombres negros y menos aún de mujeres negras en las altas funciones de la sociedad civil del Estado y de las empresas privadas, al igual que la falta de imágenes positivas de hombres y mujeres negros en los medios de comunicación y en las representaciones simbólicas de los lugares públicos no hace sino atestiguar de la persistencia del racismo en Colombia... Es necesario recordar también los limitados alcances de las políticas multiculturales en el reconocimiento de las necesidades "etno-

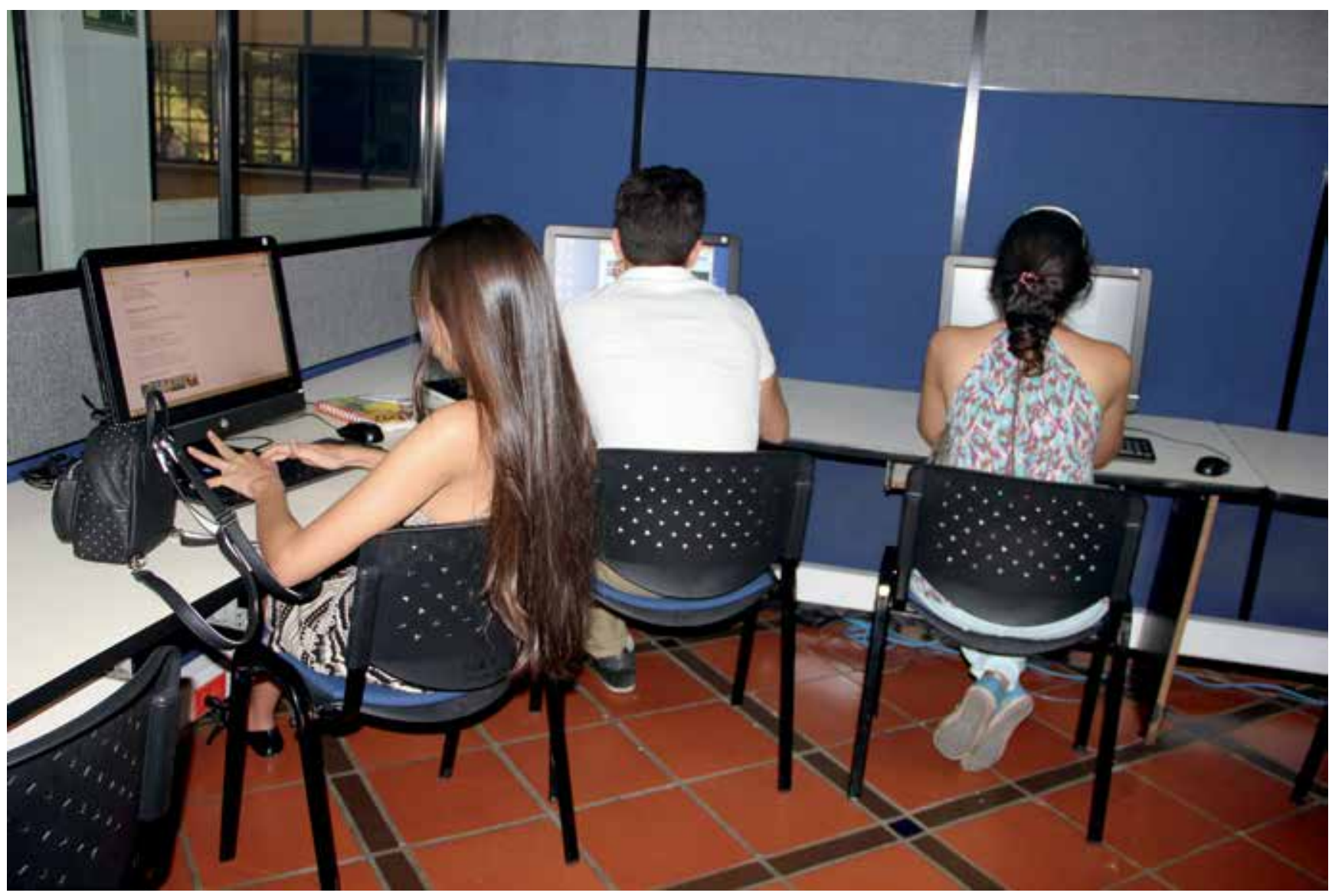


culturales" de las poblaciones indígenas y afrocolombianas y de su aporte a la historia y cultura de la nación. Igualmente son pocos los logros obtenidos a través de los fondos especiales de becas para educación superior destinados a estas poblaciones que en la práctica no significan sino un atenuante de las desigualdades que las afectan (Vigoya, 2012, pp. 10 y 11).

Sin embargo, las cifras anteriores deben analizarse considerando el número de programas y proyectos especiales que privilegian la educación inclusiva en comunidades afrodescendientes y los factores que pueden repercutir para que aún existan estas diferencias.

También se destaca la investigación realizada por Beltrán, Martínez \& Vargas (2015) en donde se realizó una comparación entre las políticas y estrategias en inclusión educativa desarrolladas en España y en Colombia, teniendo en cuenta las categorías de enfoque, grupos de atención prioritaria, responsables y recursos, estrategias de enseñanza y aprendizaje, diseño curricular, niveles educativos, formación docente, participación de la comunidad y evaluación y seguimiento; permitiendo concluir que aunque Colombia ha avanzado en el tema aún se hace necesario fortalecer muchos aspectos tales como la formación docente, el sistema de evaluación y especialmente las barreras y predisposiciones generalizadas en la sociedad.

Ahora, a pesar de los límites que pueden existir y de las múltiples barreras, se hace necesario tomar conciencia de que

Como parte de su contribución a la sociedad desde la investigación, la producción y la docencia, se espera que la Universidad favorezca la formación profesional de estudiantes que proceden de distintos estratos y presentan diferencias significativas en cuanto a sus condiciones personales y culturales (Chiny, Salas \& Vargas, 2008, p. 72).

En este mismo sentido, se destaca lo planteado por Leiva (2013) considerando que "las instituciones universitarias de educación de- ben ser motores de cambio educativo y de transformación de mentalidades sociales para la mejora y el progreso de la sociedad y la ciencia" (p.3) razón por la cual considera la necesidad de profundizar en el concepto de inclusión, generando un análisis serio y capaz de impactar en la comunidad universitaria.

\section{La educación a distancia y el concepto de inclusión}

De acuerdo con Yee \& Miranda (2006) la educación es fundamental para el desarrollo de los pueblos, por eso se hace necesario que esta sea inclusiva, abordando todos los mecanismos necesarios para serlo, a través de nuevos enfoques y alternativas, entre las que se destaca de forma fundamental la educación a distancia.

Frente a lo anterior, Bayardo (citado por Yee \& Miranda, 2006) expone que

Enseñar y aprender a distancia más allá de los antagonismos creados el siglo pasado, debe pasar a ser parte fundamental de la educación en cualquier sociedad pues puede cubrir algunos huecos que la educación tradicional no ha podido cubrir, por ejemplo el rezago educativo de los adultos, y la atención a varios grupos diversificados que tienen hábitos y costumbres propios como los grupos indígenas, grupos de escasos recursos y personas discapacitadas entre otros (p. 186).

De esta manera, la educación a distancia se relaciona con la inclusión, puesto que brinda posibilidades a aquellos que se les dificulta acceder a la tradicional por razones geográficas, de edad, ocupación, horario, entre otras, brindando independencia y autonomía en el estudio y un acceso de forma equitativa (López \& Pedraza, 2012).

Así mismo, el Comité de Derechos Económicos, Sociales y Culturales de las Naciones Unidas CDESC (2008) define que el Estado debe garantizar los derechos a la educación teniendo en cuenta cuatro dimensiones: Disponibilidad, aceptabilidad, adaptabilidad y accesibili- 
dad. Esta última, la plantea en cuanto a la no discriminación, exponiendo que "la educación ha de ser asequible materialmente, ya sea por su localización geográfica de acceso razonable (por ejemplo, una escuela vecinal) o por medio de la tecnología moderna (mediante el acceso a programas de educación a distancia)" (Unesco, 2008, p. 28).

Así mismo, al hablar de la relación entre educación a distancia e inclusión, Vizcaíno \& Orozco (2008) plantean que otras ventajas están en el hecho de poder trabajar y estudiar al tiempo, generando una mejora en los ingresos y en la accesibilidad sin necesidad de grandes desplazamientos.

También, García \& Moreno (2012) al hablar de las prospectivas de la modalidad a distancia exponen que esta debe redefinir la educación teniendo en cuenta que los ambientes de aprendizaje deberán permitir la inclusión social, para lo cual toda la comunidad educativa deberá estar involucrada.

En este sentido, varios autores han coincidido que una herramienta fundamental para la educación a distancia y que le permite ser inclusiva radica principalmente en el uso de las TIC, por lo cual:

La configuración de contextos de inclusión social considera todas las formas de inserción social, para todos los usuarios (estudiantes, grupos, comunidad, entre otros), con herramientas para el ejercicio de competencias digitales, a fin de desenvolverse con pertinencia y prudencia, de manera proactiva y responsable, en una (ciber) ciudadanía, para comportamientos valiosos, productivos y válidos que satisfagan sus necesidades de vida (Fainholc, 2010, p. 3).

Este aporte de las nuevas tecnologías, ya venía siendo planteado desde antes, tal como destaca Pastor (2005) en donde analiza su importancia para la educación a distancia en América Latina, destacando que se convierte en un nuevo paradigma y propicia un aprendizaje significativo e innovador.
Así mismo, se plantea que "los programas de educación electrónica y las TIC en educación no garantizan por sí mismos la inclusión y la equidad social, ni la calidad e innovación educativas" (Fainholc, 2010, p. 7) y que por lo tanto se hace necesario renovar el paradigma educativo, fortalecer el sistema investigativo, académico y la infraestructura y analizar las debilidades del proceso.

Tales dificultades en la inclusión se pueden encontrar en los diferentes sectores de la comunidad educativa, incluyendo el mismo estudiantado. En una investigación realizada por Novo \& Muñoz (2012) sobre la forma en que los estudiantes asumían las diferencias de sus compañeros, se detectaron buenas actitudes hacia estos; sin embargo, pocos se involucran en el proceso; en el estudio también se ratifica a la educación a distancia como una excelente opción para los estudiantes con dificultades.

Por lo tanto, es necesario considerar las barreras pero también los facilitadores para la aplicación de las políticas de inclusión educativa del discapacitado, tal como lo expone Serrano \& Camargo (2010) identificando la falta de estrategias, los altos costos, la escasa formación del profesorado y las actitudes negativas; aunque también se encuentra la disponibilidad de cupos y el interés de las familias.

En cuanto a las barreras y dificultades Palomero (2006) por medio de un estudio de caso en Aragón analiza la formación de los docentes en cuanto a la inclusión intercultural, encontrando poca importancia a los problemas educativos que se derivan de la inmigración, desplazamiento y la participación de diversas culturas; para lo cual propone una nueva pedagogía con un nuevo plan de estudios.

En el mismo sentido, Navarrete, Candia \& Puchi (2013) destacan la experiencia vivida en Perú con la Universidad de la Frontera, que ha permitido brindar educación superior a los indígenas, pero la barrera que se evidencia ha 
sido la deficiencia en el rendimiento, puesto que sus notas suelen ser inferiores a la de sus compañeros, lo cual se convierte en un motivo de exclusión y deserción.

Salinas et al. (2013) también plantean que para el proceso de inclusión en educación superior se presentan facilitadores y algunas barreras, entre los primeros se destaca el respeto hacia la diferencia, el apoyo del entorno, especialmente de la familia; pero en las limitaciones está la falta de conocimiento sobre discapacidad y de comprensión de las diversas necesidades educativas.

No obstante, la educación a distancia a través de sus diversas herramientas, entre las que se destaca el uso de las TIC, se considera pieza clave para la inclusión. En este sentido, Palou \& Antonetti (2010) plantearon la formación de un grupo de reclusos de la localidad de Río de Cuarto, Córdoba, Argentina, en una carrera de nivel superior (Técnico en Administración de Empresas a distancia), buscando dar herramientas de reinserción social una vez terminada su condena; y así obtener gran impacto y logros significativos, al resaltar como herramientas el seguimiento tutorial y la formación ofrecida.

En este mismo enfoque de la virtualidad se realizó en México un proyecto sobre inclusión educativa virtual de estudiantes con discapacidad motora, partiendo de la educación básica hasta llevarlos a la superior, sin embargo, se encontraron dificultades como la falta de herramientas tecnológicas y el manejo de estas, además el cansancio muscular por los movimientos repetitivos, destacándose que al momento de realizar inclusión se hace necesario adaptarse a las diversas realidades de los individuos, sin pretender que sean ellos quienes los hagan (Camacho, Varela \& Gerardo, 2011).

Por su parte, Solari \& Germán (2005) también observaron dificultades en el uso de las tecnologías, llegando a considerar que pueden convertirse en una forma de segregación y exclu- sión entre los que pueden y saben usarla y los que no; por lo cual se invita a las instituciones de educación superior a superar dichos límites, propiciando la inclusión.

Sin embargo, la virtualidad continúa siendo un factor indispensable que propicia la inclusión. Desde ese punto de vista Rama (2013) analiza el acceso a la educación superior de personas con discapacidad, manifestando que las dificultades se incrementa si se continúa con los métodos tradicionales, por lo cual plantea que

El aprendizaje colaborativo en red y el hogar como aula digital, se constituyen en la única modalidad que efectivamente permite la total accesibilidad para todas las personas con discapacidad, más allá de la diversidad de situaciones y patologías, y analizar como bajo el mundo digital no hay personas con discapacidades en tanto los soft y los hardware se constituyen en los interfaces de la accesibilidad a la educación (p. 326).

También Arboleda (2013) expone que la virtualidad educativa permite potenciar una educación con calidad y pertinencia favoreciendo la inclusión de aquellos estudiantes que presenta limitaciones geográficas, tecnológicas y culturales.

Así mismo, Rojas (2013) realizó una investigación con el propósito de analizar el papel de la educación a distancia (EaD) como factor de inclusión social a partir de la experiencia de la UNED Costa Rica y de la UAPA República Dominicana, y observó las acciones relacionadas con la docencia, investigación y extensión que se ejecutan en miras de la inclusión educativa y tomó en cuenta factores como el acceso, la calidad y la igualdad de oportunidades. Dicho proyecto se encuentra en curso por lo que aún no se pueden establecer conclusiones sobre el mismo.

Por su parte, Pagano (2007) manifiesta que para un adecuado proceso de inclusión en la educación a distancia es fundamental la figura del tutor, quien potencia el aprendizaje de sus estudiantes y sirve de apoyo en aquellas actividades que se le dificulta al alumno, teniendo en 
cuenta a los que presentan deficiencias en su aprendizaje individual.

Dentro de esa perspectiva se destaca también la necesidad de cultivar algunas habilidades fundamentales en los docentes tales como las planteadas por Fernández (2013) en cuanto a ser tutor, tener habilidades comunicativas y usar metodologías y materiales acordes a los contextos.

En México, Alcántara \& Navarrete (2014) destacan la creación de una universidad intercultural que busca la formación de profesionales comprometidos con la sociedad a partir de la investigación sobre cultura e idiomas. Sin embargo, se plantea varios desafíos principalmente en calidad educativa y en el hecho de que aun persista discriminación, exponiendo como posibles causas que

está muy enraizada en las estructuras sociales, políticas, económicas, culturales, jurídicas, estéticas, etcétera, y esto exige conocer, visibilizar, mapear, graficar y relacionar esas estructuras para dimensionar la magnitud de los problemas alrededor de la discriminación, desentrañar la trama de sus interrelaciones y así poder contar con herramientas idóneas para diseñar y definir políticas públicas incluyentes que atajen efectivamente las injusticias de la exclusión (Raphael, citado por, Alcántara \& Navarrete, 2014, p. 234).

La discriminación, por lo tanto, se convierte en un obstáculo ante la inclusión educativa, tal como lo expresa Chávez (2008), quien realizó una investigación acerca de los problemas que enfrentaba una comunidad indígena en la Universidad Autónoma de Chapingo, permitiendo establecer el aislamiento al que se enfrentan los estudiantes y las estrategias que se deben usar para garantizar su permanencia.

También en México Zambrano, Hernández \& Ramírez (2009) realizan una investigación sobre la inclusión social en experiencias de evaluación de entornos virtuales, encontrando que es posible si se suscitan espacios de interacción entre los estudiantes y docentes, como la creación de comunidades académicas basadas en aprendizaje colaborativo y en el uso de las nuevas tecnologías.

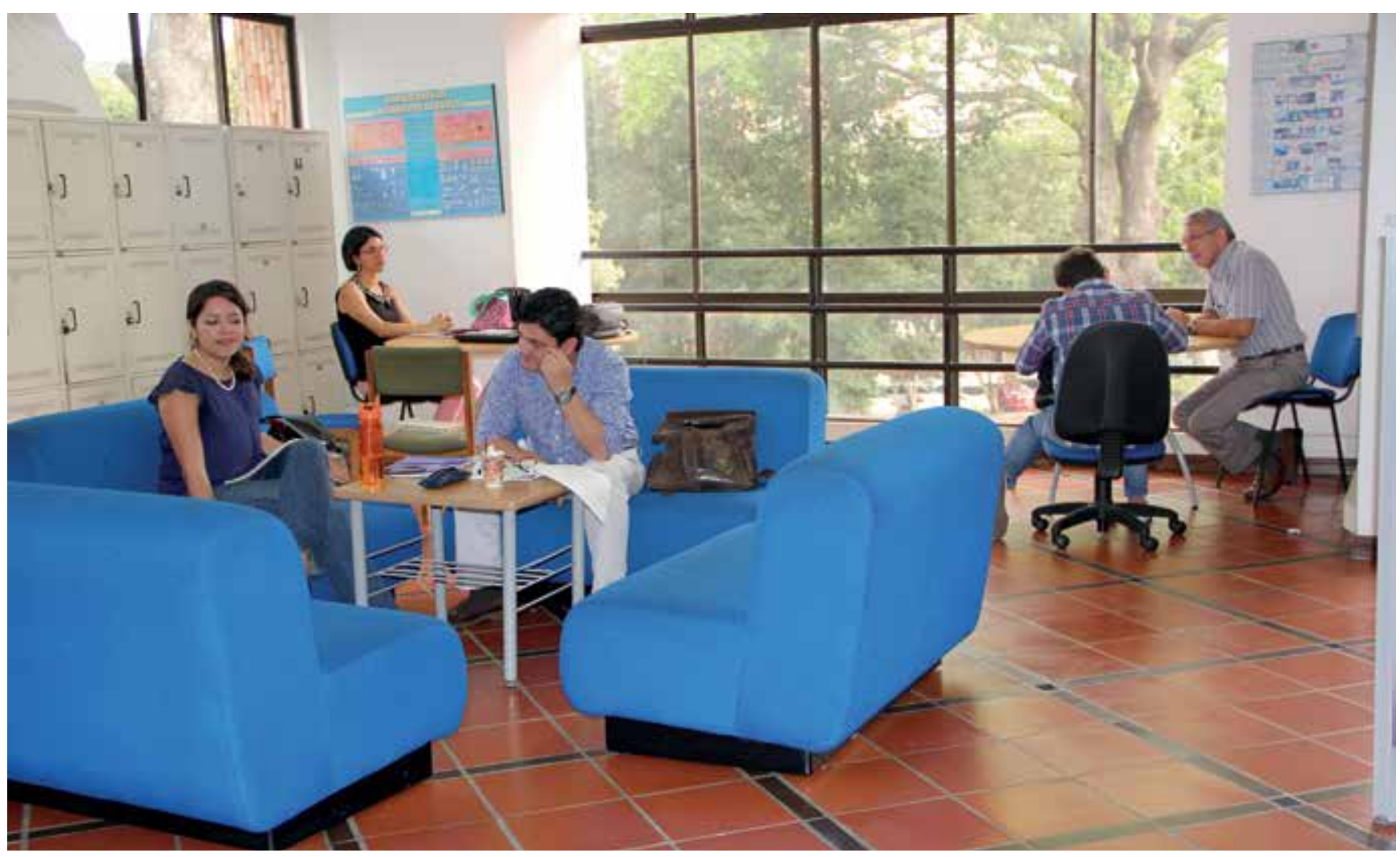


Similar a lo anterior, Pérez \& Sarrete (2013) analizan la diversidad cultural y el papel de las instituciones de educación superior en la inclusión social, al destacar sus limitaciones y ventajas y elaborar propuestas que promuevan el respeto por las diferencias desde la modalidad a distancia.

Por su parte, en Colombia, la Fundación Universitaria Católica del Norte (2013) creó un centro de inclusión en búsqueda de fortalecer el acceso a la educación por medio del uso de las tecnologías de la información y la comunicación, realizando "acciones de atención, asesoría, capacitación, formación e investigación en todos los campos de la inclusión educativa y social de las personas con discapacidad, o con condiciones de vulnerabilidad" (p. 7).

En este sentido, Martínez (2011) muestra cuatro experiencias significativas de inclusión educativa, utilizando la modalidad a distancia con ayuda de las tecnologías. En primer lugar, se destaca la plataforma Tiresias desarrollada por la Universidad de Antioquia para el apoyo a los estudiantes con discapacidad visual, contando con material audiovisual y documentación bibliográfica en braille. Además, con un comité de inclusión que propone y regula las políticas y estrategias para el acceso a la educación de la población en condiciones de discapacidad.

La segunda estrategia que se destaca es en la Universidad de San Buenaventura seccional Medellín, quien cuenta con el programa InclUtics y el recurso tecnológico como Plaphoons, teclado virtual, Zacbrowse, ratón facial, entre otros, para garantizar el acceso a la educación de personas con discapacidad visual, auditiva y motora. Además, el proyecto de alfabetización digital en el resguardo de Guambia (Cauca, Colombia) en donde se tradujo a la lengua nativa algunos términos relacionados con el uso de las TIC, obteniendo un material multimedia según las costumbres y cultura de la comunidad (Martínez, 2011).

De igual forma, en Colombia se destaca el estudio realizado por López \& Pedraza (2012) sobre la inclusión y la lectura y la escritura a

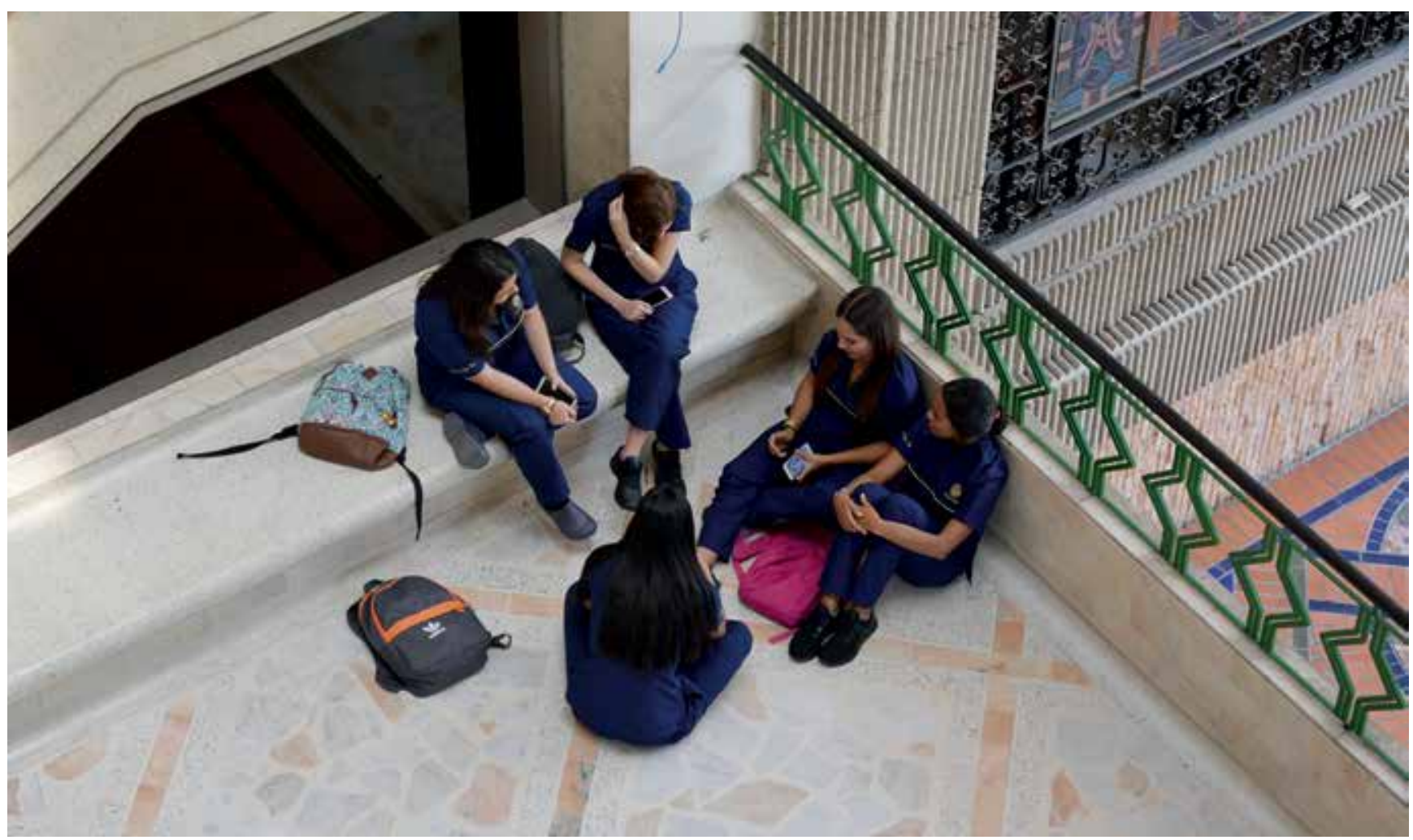


través de las TIC en la educación superior a distancia, donde a partir de estas habilidades básicas se propicia la vinculación de los estudiantes y se mejoran sus competencias con procesos de investigación, revisiones bibliográficas, consultas, escritos, elaboración de resúmenes, entre otros.

Así mismo, Hernández, Pedraza \& López (2011) crean un dispositivo tecnológico para la optimización del tiempo de aprendizaje del lenguaje braille en personas invidentes, en el que a partir de un signo se genera la voz de manera artificial y se reproduce una señal audible, facilitando el proceso de escritura y la velocidad de digitación.

\section{Conclusiones}

Aunque al realizar una revisión de la bibliografía se encuentran diversas investigaciones especialmente en lo relacionado con inclusión y discapacidad y se han creado políticas y estrategias, aún queda mucho por hacer en lo relacionado con el acceso y calidad educativa.

En este sentido, Villaran \& Guerrero (2008) plantean que se requiere apuntar a la salud individual, al aprendizaje, vinculación laboral, y a eventos de violencias, embarazos adolescente, conflicto armado, entre otras. Se hace necesario, por lo tanto, ir más allá en materia de inclusión, donde las instituciones educativas aparte de contemplar en su PEI una política creen estrategias eficaces y toda la organización fomente una cultura inclusiva donde haya espacios para todos, sin excepción.

Así mismo, Juárez, Comboni \& Garnique (2010) exponen como retos para avanzar en una educación inclusiva la necesidad de cambios en las políticas y los sistemas educativos, en las actividades y prácticas, donde se valore la diversidad, se garantice la calidad, se comprometa con el cambio y se revalore la función docente.

También, Miklos \& Arroyo (2008) en una visión prospectiva de la educación a distancia en
América Latina plantean la necesidad de proyectarse a nivel de equidad, autogestión, convergencia, diversidad, integración, colaboración y cooperación para propiciar un adecuado proceso de inclusión.

Es necesario considerar que aunque el Ministerio ha priorizado a poblaciones como desplazados, discapacitados, mujeres, afrodescendientes, habitantes de fronteras, también se requiere adoptar políticas para otro tipo de poblaciones vulnerables, tales como: la comunidad LGTBI, madres solteras o cualquier individuo cuyos derechos sean vulnerados y que viven ciertas discriminaciones.

Por lo tanto, es urgente una educación incluyente, tal como lo plantea Martínez (2011), donde se promueva el respeto por las diferencias, se garantice la igualdad de oportunidades, el diálogo intercultural, el respeto y la tolerancia y cuya prioridad sea escuchar al otro, dándole el lugar que necesita para desarrollarse y ser parte de la sociedad.

En dicho proceso es necesario que se involucre toda la comunidad universitaria, al ir más allá de las estrategias y modelos tradicionales, y propiciar que los estudiantes y docentes acojan a los individuos aunque tengan diversas costumbres y hábitos, aprendiendo a convivir, respetar y aceptar las diferencias, como una opción por los vulnerables.

Finalmente, se requiere considerar que la inclusión es un asunto de todos y por lo tanto, es necesario continuar buscando metodologías y estrategias que logren nuevas actitudes hacia la población con necesidades especiales y la generación de procesos que puedan garantizar unos servicios educativos con calidad, donde cada individuo sea entendido en la totalidad de su ser.

\section{Referencias}

Alcántara, A., y Navarrete, Z. (2014). Políticas de inclusión a estudiantes indígenas en la educación superior. Recuperado de http://www.ridaa.es/ridaa/index.php/ ridaa/article/view/110/107. 
Alcántara, A., \& Navarrete, Z. (2014). Inclusión, equidad y cohesión social en las políticas de educación superior en México. Recuperado de http://www.redalyc.org/ pdf/140/14029405010.pdf

Arboleda, N. (2013). La nueva relación entre tecnología, conocimiento y formación tiende a integrar las modalidades educativas. Recuperado de http://virtualeduca.org/documentos/observatorio/la_educacion_ superior_a_distancia_y_virtual_en_colombia_nuevas_realidades.pdf

Beltrán, Y., Martínez, Y., y Vargas, A. (2015). El sistema educativo colombiano en el camino hacia la inclusión. Avances y retos. Recuperado de http://www.redalyc. org/pdf/834/83439194004.pdf

Camacho, C., Varela, N., y Geraldo, A. (2011). Inclusión educativa virtual de estudiantes con discapacidad motora. Recuperado de http://www.redalyc.org/ pdf/688/68826916005.pdf

Chávez, M. (2008). Ser indígena en la educación superior ¿desventajas reales o asignadas? Recuperado de http://www.scielo.org.mx/scielo.php?script=sci_artt ext\&pid=S0185-27602008000400003

Chiny, J., Salas, K., y Vargas, M. (2008). Accesibilidad para ingresar a la educación superior: desafíos y logros desde el enfoque de la diversidad. Recuperado de http:// www.redalyc.org/pdf/1941/194114582011.pdf

Chiroleu, A. (2009). La inclusión en la educación superior como tema de la agenda de gobierno en América Latina. Una reflexión sobre las propuestas del CRES/2008. Recuperado de http://www.redalyc.org/ pdf/373/37313028003.pdf

Chiroleu, A. (2009). Políticas públicas de inclusión en la educación superior. Los casos de Argentina y Brasil. Recuperado de http://www.scielo.br/pdf/pp/v20n2/ v20n2a10

Chiroleu, A. (2012). Políticas públicas de educación superior en América Latina: ¿democratización o expansión de las oportunidades en el nivel superior? Recuperado de http://www.redalyc.org/html/122/12226914006/ index.html

Dueñas, M. (2010). Educación inclusiva. Recuperado de http://www.redalyc.org/articulo.oa?id=338230785016

Fainholc, B. (2010). Hacia la configuración de la inclusión social y la consolidación de la convivencialidad con el aporte de la educación virtual. Recuperado de http:// www.redalyc.org/pdf/688/68813176004.pdf
Fernández, J. (2013). Competencias docentes y educación inclusiva. Recuperado de http://redie.uabc.mx/redie/ article/view/445/610

Fundación Universitaria Católica del Norte. (2013). Configuración y fundamentación del centro de estudios en inclusión. Recuperado de http://www.ucn.edu.co/ sistema-investigacion/Documents/sistema-investigacion-innovacion/FundamentacionConceptualCentrosEstudio/CentroEstudiosInclusion.pdf

García, A. (2014). Inclusión social en la educación superior argentina: Indicadores y políticas en torno al acceso y a la graduación. Recuperado de http://www.cedes.org/ publicaciones/documentos/EdSup/2014/10669.pdf

García, M., y Moreno, M. (2012). Prospectiva de la educación a distancia. (Cap. 7). Recuperado de http://biblioteca.udgvirtual.udg.mx/eureka/pudgvirtual/Encuentro_web.pdf

Hernández, C., Pedraza, L., y López, D. (2011). Dispositivo tecnológico para la optimización del tiempo de aprendizaje del lenguaje Braille en personas invidentes. Recuperado de http://www.redalyc.org/ pdf/422/42222409015.pdf

Infante,M.(2010). Desafíosalaformacióndocente:Inclusión educativa. Recuperado de http://www.scielo.cl/scielo.php?pid=S0718-07052010000100016\&script $=$ sci arttext.

Juárez, J., Comboni, S., y Garnique, F. (2010). Dossier: Procesos educativos en América Latina: política, mercado y sociedad. De la educación especial a la educación inclusiva. Recuperado de http://www. scielo.org.mx/scielo.php?script=sci_arttext\&pid $=$ S0187-57952010000100003

Leiva, J. (2013). De la integración a la inclusión: evolución y cambio en la mentalidad del alumnado universitario de educación especial en un contexto universitario español. Recuperado de http://www.redalyc.org/ pdf/447/44729878025.pdf

López, K., y Pedraza, C. (2012). La inclusión y la lectura y la escritura a través de las TIC en la educación superior a distancia. Recuperado de http://media.utp.edu. co/referencias-bibliograficas/uploads/referencias/ ponencia/ponencia-lopez-y-pedraza-inclusion-eadredleespdf-uQOMf-articulo.pdf

Martínez, M. (2011). Experiencias de inclusión educativa en Colombia: hacia el conocimiento útil. Recuperado de http://www.redalyc.org/pdf/780/78017126003.pdf 
Mato, D. (2008). Diversidad cultural e interculturalidad en educación superior. Experiencias en América Latina. Recuperado de http://www.uv.mx/iie/files/2013/01/6Capitulo-Dietz-UVI.pdf

Miklos, T., y Arroyo, A. (2008). Una visión prospectiva de la educación a distancia en América Latina. Recuperado de http://www.redalyc.org/pdf/373/37311274005.pdf

Ministerio de Educación Nacional. (2007). Educación inclusiva con calidad: módulo $n^{\circ} 1$, procedimientos y conceptos básicos para una educación inclusiva con calidad. Bogotá: Colombia

Ministerio de Educación Nacional. (2013). Panorama de la inclusión en la educación superior en Colombia. Recuperado de http://www.mineducacion.gov.co/ cvn/1665/w3-article-327505.html

Ministerio de Educación Nacional. (2013). Lineamientos. Política de educación superior inclusiva. Recuperado de http://www.dialogoeducacionsuperior.edu. co/1750/articles-327647_documento_tres.pdf

Molina, R. (2010). Educación superior para estudiantes con discapacidad. Recuperado de http://www.urosario.edu.co/urosario_files/aa/aa7a656b-30b9-4dec87dd-6a174a507cba.pdf

Narvarte, L., y Blanco, R. (2010). Educación especial e inclusión educativa. Recuperado de http://site.ebrary. com/lib/bibliocunsp/reader.action?docID=10418389

Navarrete, S., Candia, R., y Puchi, R. (2013). Factores asociados a la deserción/retención de los estudiantes mapuche de la Universidad de la Frontera e incidencia de los programas de apoyo académico. Recuperado de http://www.scielo.cl/scielo.php?pid=S071845652013000200003\&script=sci_arttext

Novo, I., y Muñoz, J. (2012). Los estudiantes universitarios ante la inclusión de sus compañeros con discapacidad: indicadores basados en la teoría de la acción razonada para los estudios de economía y empresa en la Universidad de Coruña (España). Recuperado de http://www. redalyc.org/pdf/3382/338230791008.pdf

Padilla, A. (2011). Inclusión educativa de personas con discapacidad. Recuperado de http://www.scielo.org.co/ $\mathrm{pdf} / \mathrm{rcp} / \mathrm{v} 40 \mathrm{n} 4 / \mathrm{v} 40 \mathrm{n} 4 \mathrm{a} 07$

Pagano, C. (2008). Los tutores en la educación a distancia. Un aporte teórico. Recuperado de http://dspace.uces. edu.ar:8180/xmlui/bitstream/handle/123456789/574/ Los_tutores_en_la_educaci\% C 3\%B $3 n_{\text {_. }}$. pdf? sequence $=1$
Palomero, J. (2006). Formación inicial de los profesionales de la educación en pedagogía intercultural una asignatura pendiente. El caso de Aragón. Recuperado de http:// dialnet.unirioja.es/servlet/articulo?codigo $=2234449$

Palou, E., y Antonetti, P. (2010). La inclusión social en el sistema de educación a distancia. Recuperado de http://www.quadernsdigitals.net/index. php?accionMenu=hemeroteca.VisualizaArticulolU. visualiza\&articulo_id=10978

Pastor, M. (2005). La educación superior a distancia en el nuevo contexto tecnológico del siglo $X X$. Recuperado de http://www.redalyc.org/pdf/604/60413606.pdf

Pedroza, R., y Villalobos, G. (2009). Políticas compensatorias para la equidad de la educación superior en Argentina, Bolivia y Venezuela. Recuperado de http://www.scielo.org.mx/scielo.php?pid=S018527602009000400003\&script=sci_arttext

Pérez, G., y Sarrete, M. (2013). Diversidad cultural y ciudadanía hacia una educación superior inclusiva. Recuperado de http://www.redalyc.org/ pdf/706/70625886005.pdf

Río, N. (2015). Políticas inclusivas en la educación superior de la ciudad de México. Recuperado de http://cdhdfbeta.cdhdf.org.mx/wp-content/uploads/2015/06/Politicas-inclusivas.pdf

Rojas, R. (2013). Educación a distancia como factor de inclusión social en la Universidad Estatal a Distancia (UNED) de Costa Rica y la Universidad Abierta para Adultos (UAPA) de República Dominicana. Recuperado de http://www.kbasemiseal.org/es/search bestpractice $/ 2150$

Salinas, M., Lissi, M., Medrano, D., Zuzulich, M., y Hojas, A. (2013). La inclusión en la educación superior: desde la voz de estudiantes chilenos con discapacidad. Recuperado de http://www.rieoei.org/rie63a05.pdf

Serrano, C., y Camargo, D. (2010). Políticas de inclusión educativa del discapacitado. Barreras y facilitadores para su implementación. Recuperado de http://www. redalyc.org/pdf/120/12021452009.pdf

Solari, A., y Germán, M. (2005). Un desafío hacia el futuro: educación a distancia, nuevas tecnologías y docencia universitaria. Recuperado de http://www.seduca2. uaemex.mx/ckfinder/uploads/files/un_desafio_a_futuro_ead.pdf

Unesco. (2008). Conferencia Internacional de Educación "La educación inclusiva: El Camino hacia el futuro". Recuperado de http://www.ibe.unesco.org/fileadmin/ 
user_upload/Policy_Dialogue/48th_ICE/CONFINTED_48_Inf_2_Spanish.pdf

Villarán, V., y Guerrero, L. (2008). Educación e inclusión en la región Andina. Recuperado de http://www.redalyc. org/pdf/4136/413635248011.pdf

Viveros, M. (2012). Equidad e inclusión en la educación superior. Algunos aportes a la discusión desde la Escuela de Estudios de Género de la Universidad Nacional de Colombia. Recuperado de http://www.bdigital.unal. edu.co/7409/1/maraviversovigoya1.pdf

Vizcaíno, A., y Orozco, M. (2008). Trayectorias y experiencias en la educación a distancia. México: Universidad de Guadalajara
Yee, M., y Miranda, A. (2006). Cuba: la educación a distancia en la Universidad de la Habana. Recuperado de http:// www.redalyc.org/pdf/3314/331427205008.pdf

Zambrano, D. J., Hernández, C. A., y Ramírez, M. S. (2009). Integración de la inclusión social en experiencias de evaluación de entornos virtuales. Memorias del Congreso Internacional de Educación: Curriculum. Universidad Autónoma de Tlaxcala. Recuperado de http:// www.ruv.itesm. $m x /$ convenio/catedra/recursos/material/ci_20.pdf 